\title{
Abordagem sistêmica na caracterização da participação no processo de Crowd-Design
}

Systemic approach in the characterization of participation in the Crowd-Design process

DICKIE, Isadora; Doutora; Univille, UFPR

isadora.dickie@gmail.com

SCHMITZ, Mariana; Bacharel; Univille

tz.mariana@gmail.com

FIGUEIREDO, Luiz Fernando Gonçalves de; Doutor; UFSC

Iff@cce.ufsc.br

SANTOS, Aguinaldo dos; Doutor; UFPR

asantos@ufpr.br

\section{Resumo}

Crowd-Design refere-se a uma modalidade emergente de processo desenvolvimento de produtos e serviços que envolve diversos stakeholders em atividades de co-design através de plataformas online. Embora a participação de stakeholders seja intrínseca ao processo de Crowd-Design, ainda faltam estudos que elucidem os níveis de participação envolvidos neste processo. Assim, o objetivo deste artigo é apresentar os resultados de uma pesquisa que buscou compreender, a partir de estudos de casos ex-post-facto, quais são os níveis de participação envolvidos em duas iniciativas de Crowd-Design da plataforma innonatives.com. A Abordagem Sistêmica foi utilizada como ferramenta para analisar e apresentar os resultados. O principal resultado desta pesquisa é, portanto, a caracterização dos processos participativos do processo de Crowd-Design.

Palavras Chave: processos baseados em multidão; abordagens participativas; processo de desenvolvimento de produto.

\begin{abstract}
Crowd-Design refers to an emerging model of product and service development process that involves multiple stakeholders in co-design activities through online platforms. Although stakeholder participation is intrinsic to the Crowd-Design process, there is still a lack of studies that elucidate the levels of participation involved in this process. Thus, the purpose of this article is to present the results of a research that sought to understand, from ex-post-facto case studies, what levels of participation are involved in two Crowd-Design initiatives of the innonatives.com platform. The Systemic Approach was used as a tool to analyze and present the results. The main result of this research is, therefore, the characterization of the participatory processes of the Crowd-Design process.
\end{abstract}

Keywords: crowd-based processes; participative approaches; product development process. 


\section{Introdução e contextualização}

Devido ao avanço nas tecnologias de informação e comunicação (TICs), estratégias para o envolvimento de stakeholders em projetos de design vem ganhando novas possibilidades, principalmente em processos que ocorrem através da internet. Ambientes online, conhecidos como 'plataformas de inovação aberta' (Batistella \& Nonino, 2012), conectam organizações e pessoas de todas as partes do mundo, atuando como facilitadoras no que diz respeito ao compartilhamento de informações e execução de tarefas para a inovação e resolução de problemas (Brabham, 2008, Sharma, 2010; Brabham, 2010, De Toni et al., 2012; Chiu et al., 2014, Prpi'c et al., 2015).

O processo que ocorre nas plataformas de inovação aberta é denominado 'crowdsourcing' . Termo cunhado por Howe em 2006, sua definição mais genérica foi dada por Estellés-Arolas \& González Ladrón-de-Guevara (2012) e refere-se à terceirização de determinadas tarefas de uma organização para uma 'multidão', sob a forma de um convite aberto em um ambiente online, ao invés de designá-las a colaboradores contratados. Desde então o termo vem ganhando derivações, cujas definições são caracterizadas principalmente pelo tipo de tarefa desempenhada pela multidão. Por exemplo, 'crowdvoting' refere-se ao processo de votação online, sobre um determinado tópico, onde a 'tarefa' da multidão é votar (Beherend et al., 2011; Bannerman, 2013; Djelassi \& Decoopman, 2013). 'Crowdfunding', por sua vez, refere-se ao processo de 'financiamento coletivo', onde a multidão, através de contribuições individuais, contribui na implementação de projetos das mais variadas áreas (Djelassi \& Decoopman, 2013).

O termo 'Crowd-Design', no entanto, apareceu na literatura apenas em 2012. Dawson \& Bynghall (2012) utilizaram o termo para se referir a uma categoria de crowdsourcing que envolve 'tarefas criativas' aplicadas ao processo de desenvolvimento de produtos e serviços. Apesar de recentes, iniciativas de Crowd-Design são cada vez mais frequentes (Dickie \& Santos, 2014). Já os estudos relacionados a este tema ainda são incipientes, inclusive no Brasil. Estudos sobre participação de stakeholders em iniciativas de Crowd-Design tem focado, principalmente, em questões relacionadas (i) à motivação dos participantes (Batistella \& Nonino, 2012, Simula \& Vuori, 2012; Simula \& Ahola, 2014; Geiger \& Schader, 2014) e (ii) ao papel desempenhado por cada participante de acordo com a tarefa executada (Thuan et al., 2016). No entanto, a necessidade de caracterizar os níveis de participação que ocorrem em processos de Crowd-Design já foi apontada por estudos que enfatizam a necessidade de definir, já no planejamento da iniciativa de Crowd-Design, questões relativas às possibilidades de participação da multidão (Peng \& Ruoyu, 2011; Djelassi \& Decoopman, 2013; Thuan et al., 2016).

Nesse contexto, visando contribuir para a minimização desta lacuna, o objetivo deste artigo é apresentar os resultados de uma pesquisa que buscou compreender e categorizar, através da aplicação da Abordagem Sistêmica, os níveis de participação envolvidos no processo de CrowdDesign. Para tanto, aplicou-se o método de estudo de casos múltiplos ex-post-facto em duas iniciativas de Crowd-Design da plataforma innonatives.com.

A plataforma innonatives.com é uma plataforma de inovação aberta, utilizada para solucionar desafios de inovação através do compartilhamento de ideias, comentários e votos. Desenvolvida pelo projeto Sustainability $\mathrm{Maker}^{1}$, esta plataforma conecta pessoas e organizações

\footnotetext{
${ }^{1}$ Projeto liderado pela empresa e-concept, da Alemanha, cujo objetivo foi o desenvolvimento da plataforma
} 
que desejam propor problemas relacionados à sustentabilidade com pessoas e organizações motivadas a desenvolver soluções criativas, bem como apoiar sua implementação (innonatives, web, 2017).

Este artigo está estruturado em quatro seções, a saber: (i) bases teóricas: esta seção estabelece conexões entre: (i.i) a efetividade da aplicação da Abordagem Sistêmica como ferramenta para identificação de estruturas e relações em processos de Design; (i.ii) as abordagens participativas no processo de Design; e (i.iii) a participação no processo de CrowdDesign; (ii) procedimentos metodológicos: esta seção apresenta os procedimentos de análise dos dados coletados; (iii) resultados e discussão: esta seção apresenta os resultados obtidos e (4) considerações finais.

\section{Bases Teóricas}

\subsection{Efetividade da Abordagem Sistêmica como ferramenta no campo do Design}

Para além de focar-se na projetação de produtos físicos, o propósito da prática do design tem evoluído rumo a um entendimento sistêmico. Seu principal desafio seria desenvolver ou possibilitar o desenvolvimento de soluções a questões altamente complexas que demandam uma perspectiva ampliada do projeto (Krucken, 2008). A partir da visão holística proposta na abordagem sistêmica, as inter-relações entre as partes de um sistema podem ser evidenciadas e analisadas (Muniz \& Figueiredo, 2007), facilitando a compreensão do sistema como um todo e de sua complexidade.

Sobre a natureza desta abordagem, Fiedler-Ferrara (2005) afirma que esta complexidade não se caracterizaria pelo privilégio do global sobre a análise das partes, mas sim pela constante articulação do todo com seus componentes. O foco se projeta da solução isolada para o sistema tendo a interdependência como paradigma: "[...] quer se trate da biosfera ou de organizações humanas: qualquer fenômeno repercute no conjunto, que por sua vez, mais ou menos tarde e de forma mais ou menos intensa, acaba repercutindo na fonte do fenômeno." (Kazazian, 2005, p. 3031).

Por ser considerada uma ferramenta que proporciona a visualização e a interpretação das relações dos elementos que compõem determinado sistema, a Abordagem Sistêmica vem sendo amplamente utilizada no campo do Design (Muniz \& Figueiredo, 2007). Nela, interessa considerar tanto a relação da solução com o contexto que a circunda - stakeholders, meio ambiente, comunidade, cultura local -, quanto o sistema de inter-relação entre as etapas do processo de design em si, do início à solução. Esta segunda dinâmica é que será tratada nos estudos de caso deste artigo.

\subsection{Abordagens participativas no design}

Maneiras de incluir não-designers no processo de criação vêm sendo pensadas e experimentadas no design, com o objetivo de fazer com que o desenvolvimento de soluções promova inclusão e coesão social. Cientes de que estão lidando com problemas contemporâneos

innonatives.com, financiado pela Comunidade Europeia através do Programa LIFE (LIFE11 ENV/DE/000342). O projeto é uma iniciativa de um consórcio de organizações e universidades e visa facilitar a conexão de pessoas que podem ter um papel relevante na resolução de problemas relacionados à sustentabilidade. No Brasil, este projeto foi desenvolvido pelo Núcleo de Design e Sustentabilidade da Universidade Federal do Paraná (NDS/UFPR) e denomina-se Sustainability Maker Brazil (SuM/BR). 
e complexos, os designers não detém todas as soluções, nem deveriam impor seu ponto de vista em soluções que muito tem a ganhar com o conhecimento dos usuários. De acordo com Brown (2009), deve-se pensar em projetos que tenham o engajamento multidisciplinar de profissionais e não profissionais da área criativa, que sejam diretamente interessados em soluções para os problemas abordados.

Esta maneira de pensar a projetação de soluções acompanha a tendência atual de compartilhamento e colaboração, que para Botsman e Rogers (2011) pode ser um motor de inovação tão radical para o design quanto foi a explosão da Revolução Industrial. Thackara (2008, p. 26) chega a afirmar que "os dias do designer solitário e brilhante estão contados". Este impacto é encarado de maneira bastante positiva numa perspectiva guiada a um futuro próximo. Sobre ele, Manzini (2008, p. 98) afirma que "uma nova atividade de design está emergindo, convidando os designers a exercerem um novo e fascinante papel. Aceitá-lo significa reconhecer positivamente que não é mais possível manter um monopólio sobre o design." Por isso, é ideal que designers trabalhem em conjunto com os stakeholders para ajustar os seus conhecimentos à realidade do contexto, podendo atuar em diferentes papéis; ora criador, ora facilitador, ora ativista; de acordo com a necessidade do projeto (Muzake \& Velásquez, 2012; Manzini, 2015).

Numa visão geral dos níveis de participação de pessoas não-designers no projeto para cocriação, Kaulio (1998) e Boukhris et. al. (2017) separam a intensidade do envolvimento deles no processo: se a solução é pensada para eles (a), com eles (b) ou por eles (c). Destes três níveis de intensidade da participação, os usuários atuariam: (a) como fonte de informação, para obtenção de dados específicos ou quando são consultados pelos designers; (b) como co-desenvolvedores, quando elaboram junto com os designers exteriorizando suas necessidades e dando opiniões; (c) como inovadores, quando podem desenhar ativamente suas próprias soluções.

Em uma bibliografia não tão recente, porém fundamental para o entendimento de processos participativos, Bordenave (1983) separa os níveis de participação de maneira mais detalhada de acordo com o nível de controle na tomada de decisão. Os níveis sugeridos por este autor foram adaptados ao processo de participação no design e estão representados na figura 01, seguido de sua explicação.

Figura 01 - Graus que a participação pode alcançar referente ao controle das decisões

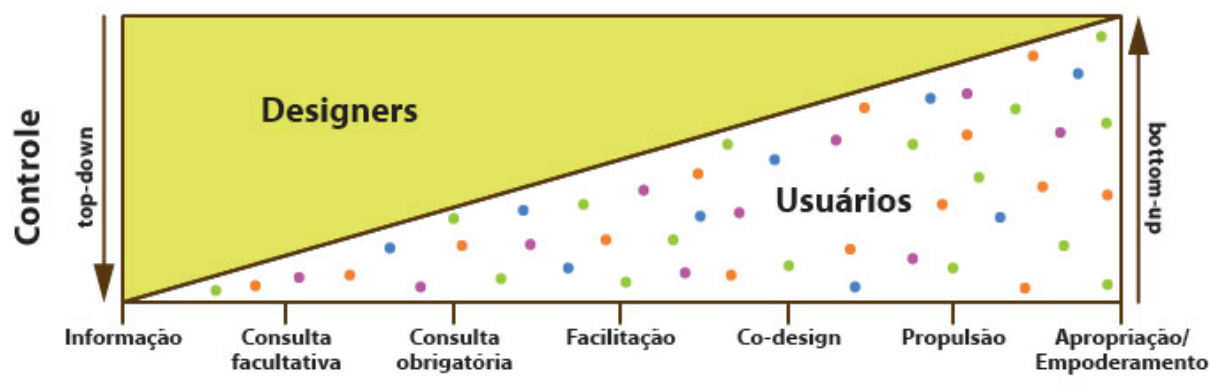

Fonte: Dos autores (2018), adaptado de Bordenave (1983).

Cada um destes níveis de participação pode ser entendido como:

- Informação: os designers informam aos usuários sobre decisões já tomadas. Este nível refere-se ao que Sanders et al. (2010) chamam de relação 'designers' e 'clientes'; 
- Consulta facultativa: solicitação de dados, críticas ou sugestões para resolver um problema, se e quando os designers desejam;

- Consulta obrigatória: os usuários devem ser consultados em certas ocasiões, mas a decisão final ainda é dos designers;

- Facilitação: usuários, por meio de mediação pelos designers, elaboram propostas e/ou recomendam medidas que podem ser aceitas ou rejeitadas no processo de criação;

- Co-design: a tomada de decisão no processo de criação é compartilhada por meio de mecanismos de co-decisão. De Toni et al. (2012) relacionam este processo à descentralização e à integração de competências;

- Propulsão: designers atuam como propulsores da autonomia dos usuários. Essa autonomia é geralmente dada por toolkits elaborados pelos designers, como por exemplo os materiais e cursos online disponibilizados gratuitamente pela IDEO.org ${ }^{2}$ sobre Human Centered Design.

- Apropriação/Empoderamento: o grupo de usuários determina seus objetivos, escolhe seus meios e toma as decisões pertinentes por si. Um exemplo pode ser encontrado no Open Design (Cabeza \& Moura, 2014).

Observa-se, ainda de acordo com a figura 01, que os níveis de participação propostos - da Informação ao Empoderamento - evoluem de uma abordagem top-down, na qual os designers ou gestores do projeto tem autoridade total na tomada de decisões, para uma abordagem bottomup, quando os usuários se apropriam integralmente do desenvolvimento da solução. A seguir, caracteriza-se o processo de participação nos projetos de Crowd-Design.

\subsection{A participação no processo de Crowd-Design}

Iniciativas de Crowd-Design envolvem processos participativos por definição. De acordo com Dickie \& Santos (2014), Crowd-Design refere-se a uma modalidade emergente de sistema de projeto e produção que utiliza os conhecimentos e recursos disponíveis na multidão, geralmente através da internet, com o propósito de resolver problemas e/ou criar conteúdo. A multidão, neste caso, refere-se a pessoas de qualquer parte do mundo que, conectadas através da internet por uma plataforma de inovação aberta, contribuem para o desenvolvimento de soluções a problemas, realizando tarefas específicas. Estas tarefas estão relacionadas a geração de conteúdo criativo (Geiger et al., 2011), como também ao compartilhamento de informações através de comentários e votos (Bannerman, 2013; Djelassi \& Decoopman, 2013; Estellés-Arolas, 2016). As possibilidades de participação em um processo de Crowd-Design são inúmeras. $E$, de acordo com a definição anteriormente apresentada, a participação pode ocorrer também fora da internet, em tarefas aqui denominadas de 'offline'.

Como visto na seção anterior, a participação em projetos de design pode ser classificada de acordo com o tipo de envolvimento dos diversos stakeholders. Em uma iniciativa de Crowd-Design há, basicamente, três stakeholders que podem ser considerados fixos (Nakatsu et al., 2014; Choi \& Lee, 2015): o ambiente virtual; o proponente da iniciativa ; e o resolutor de tarefas, onde:

\footnotetext{
${ }^{2}$ IDEO é uma empresa internacional de consultoria em Design e inovação fundada em 1991 em Palo Alto, California. Dela, surgiram iniciativas como a IDEO.org - braço da organização que trabalha com design de impacto social, divulgando o método Human Centered Design (IDEO, web, 2017).
} 
- Ambiente virtual: $\mathrm{O}$ ambiente virtual refere-se à plataforma online onde a iniciativa de Crowd-Design ocorrerá. Há pelo menos duas possibilidades neste caso: (a) a plataforma pertencer à própria organização que inicia o processo de Crowd-Design, ou (b) a plataforma atuar como facilitadora, ou agente da iniciativa (Gassmann et al., 2010; Stanoevska-Slabeva, 2011).

- Proponente da iniciativa: O proponente da iniciativa, como o nome já diz, refere-se a quem inicia o processo de Crowd-Design. Indivíduos da multidão e/ou organizações podem iniciá-lo a partir de uma demanda ou necessidade. Geralmente, essas iniciativas são chamadas de 'desafios' (Shoyama et al., 2014) e são postados pelos proponentes no ambiente virtual em forma de briefing. Cabe ao proponente da iniciativa, portanto, o planejamento das etapas do processo, bem como o resultado esperado de cada uma delas e do processo como um todo (Thuan et al., 2016).

- Resolutor de tarefas: Resolutores de tarefas são aqueles que, de fato, participam das iniciativas e desempenham ao menos uma tarefa (podendo esta ser criativa ou não) durante o processo de desenvolvimento da solução (Choi \& Lee, 2015). Assim como ocorre com o proponente da iniciativa, os resolutores de tarefas também podem ser indivíduos da multidão e/ou representantes de organizações. As características de cada resolutor, porém, está associada ao tipo de tarefa que precisa ser realizada (Geiger et al., 2011).

Ainda, é importante destacar que as iniciativas de Crowd-Design, independente do tipo de plataforma em que ocorrem, podem envolver stakeholders pré-definidos. Ou seja, o processo continua sendo realizado através da internet, porém, a 'chamada aberta' é direcionada a um determinado público com características pré-definidas (Geiger et al., 2011). De acordo com Geiger et al. (2011), há quatro possibilidades com relação ao resolutor da tarefa: (i) não pré-selecionar os participantes, (ii) pré-selecioná-los de acordo com (ii.a) sua qualificação (ou seja, habilidades e conhecimentos específicos) e (ii.b) com o contexto específico em que se encontram (por exemplo, funcionários de uma empresa); e (iii) pré-selecioná-los de acordo com a combinação das duas anteriores. Assim, resolutores considerados em 'contexto específico' podem ser associados, ainda, a iniciativas de 'Crowd-Design interno' (Simula \& Ahola, 2014) e continuam sendo consideradas casos de inovação aberta (Stanoevska-Slabeva, 2011; Tischner \& Beste, 2017).

Os três stakeholders fixos podem assumir diferentes papéis nas iniciativas de CrowdDesign, dentre elas: (i) patrocinador; (ii) especialista; (iii) criativo; (iv) votante; (v) comentarista; and (vi) gerente. Além destes papéis, é importante prever um outro: o regulamentador. De acordo com Bannerman (2013) e Brabham (2013), não apenas pelo fato de haver questões relacionadas à propriedade intelectual, mas também aos chamados 'trabalhos especulativos', algumas iniciativas estão ajudando a promover práticas éticas e profissionais nas iniciativas de Crowd-Design. Isto é, estas iniciativas estão promovendo políticas de boas práticas relacionadas ao processo de CrowdDesign.

Organizações e indivíduos da multidão podem assumir mais de um papel durante o processo de Crowd-Design. Ao mesmo tempo, quase todos os papéis podem ser assumidos por organizações e indivíduos, exceto o de 'gerente' e o de 'regulamentador'. No entanto, de acordo com Battistella \& Nonino (2012), a multidão pode estar envolvida em praticamente todas as etapas do processo de desenvolvimento de produtos, desde o levantamento de problemas ao desenvolvimento de ideias e soluções. 
Geralmente, cada plataforma online nomeia o envolvimento dos stakeholders de maneiras diferentes. Por exemplo, o 'proponente' é denominado 'seeker' na plataforma innonatives.com e 'patrocinador' na plataforma Open IDEO. Nesta última plataforma, indivíduos da multidão não podem iniciar desafios, apenas organizações. Por este motivo, o 'proponente' é chamado de 'patrocinador'. Em outros casos, como na plataforma Lego Idea, o 'votante' é denominado 'apoiador'. Ainda nesta plataforma, a ideia enviada tem que ter mais de dez mil apoiadores para ser qualificada e revisada pelos especialistas da empresa (Lego Ideas, web, 2017).

Específico da plataforma Open IDEO, há um outro papel, considerado indireto: 'personas', e se refere às pessoas para quem as soluções são destinadas (Open IDEO, web, 2017). Neste caso, a participação ocorre de maneira indireta e offline.

De acordo com Djelassi \& Decoopman (2013), cada iniciativa de Crowd-Design deve especificar formalmente o grau e a natureza da participação da multidão. Esta especificação, segundo as autoras, auxilia a recrutar a multidão e a motivá-la na participação de iniciativas de Crowd-Design. Nesse sentido, esclarecer a estratégia, bem como caracterizar a participação neste tipo de processo torna-se fundamental para o sucesso da iniciativa.

\section{Procedimentos metodológicos}

Os casos analisados neste estudo referem-se a duas iniciativas de Crowd-Design da plataforma innonatives.com, a saber: 'The Kitchen Challenge' e 'Water for Life'. Ambas iniciativas foram investigadas na pesquisa de doutorado da principal autora deste artigo, e aplicadas pela mesma nos anos de 2014 e 2015, respectivamente. O projeto Sustainability Maker (SuM, web, 2017) propõe a seguinte estruturação do processo de Crowd-Design (figura 03, página seguinte) aplicado à plataforma innonatives.com: problemas relacionados à sustentabilidade; crowdvoting; desafio; crowdsourcing; soluções; crowdvoting; painel de especialistas; melhor solução; crowdfunding, marketplace e leilões; implementação.

Assim, 'problemas relacionados à sustentabilidade', no processo da plataforma innonatives.com, refere-se à definição de uma necessidade que precisa ser solucionada pela multidão. Nesse sentido, tanto indivíduos da multidão como organizações podem iniciar o levantamento de problemas através da abertura de um desafio na plataforma. Após a sugestão dos problemas, a multidão pode escolher qual deles será solucionado, através do procedimento de votação online, o crowdvoting.

Uma vez determinada a necessidade, uma nova etapa se inicia: a de solução a esta necessidade. A plataforma innonatives.com permite que os desafios possuam até três fases para as tarefas criativas, a saber: 'envio de ideias'; 'envio de conceitos' e 'envio de solução'. De acordo com a estruturação apresentada na figura 03, estas fases referem-se ao crowdsourcing. Enquanto da duração de cada uma destas fases, outras tarefas podem ser desempenhadas, como por exemplo, envio de comentários e votação para a escolha da melhor ideia, conceito e solução. Para a escolha da melhor solução, porém, entram em cena outros stakeholders: os especialistas. Estes, na plataforma innonatives.com referem-se aos membros que possuem conhecimentos em Design para a Sustentabilidade. A atuação dos especialistas, neste caso, serve para avaliar a solução em termos de suas contribuições para a sustentabilidade. 
Figura 03 - Processo de Crowd-Design do projeto Sustainability Maker.

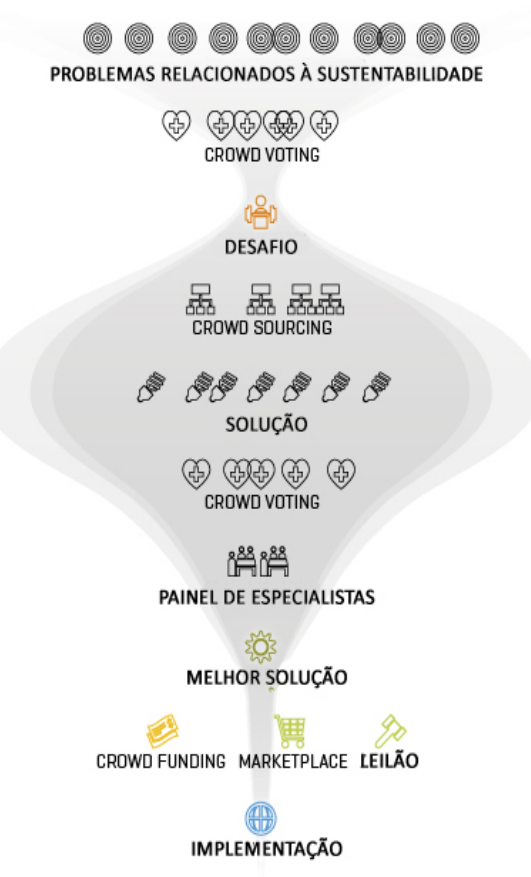

Fonte: Adaptado de SuM (2018).

Após a escolha da melhor solução - não somente pelos especialistas, mas pela multidão em geral, a plataforma innonatives.com permite que a solução seja implementada através de processos como crowdfunding, marketplace ou leilões.

No que se refere aos dois casos deste estudo, as análises foram realizadas de acordo com a comparação do processo de Crowd-Design da plataforma innonatives.com, a: (a) as particularidades do processo de Crowd-Design, adaptado de acordo com as necessidades de cada caso e (b) os níveis de participação envolvidas no processo. Nesse sentido, a Abordagem Sistêmica foi aplicada como ferramenta, proporcionando uma visão holística das iniciativas, bem como o entendimento da participação dos stakeholders envolvidos.

\section{Resultados e discussão}

\subsection{Caso 1: "The Kitchen Challenge"}

O primeiro caso analisado foi o "The Kitchen Challenge"; iniciativa de Crowd-Design vinculada ao projeto Sustainability Maker Brazil, desenvolvido por uma equipe de pesquisadores do Núcleo de Design e Sustentabilidade da Universidade Federal do Paraná (NDS/UFPR). O processo de Crowd-Design desta iniciativa teve início com a elucidação do problema a ser solucionado. Visando encontrar necessidades reais da população de uma comunidade de baixa renda localizada em Piraquara - PR, as estratégias de envolvimento da comunidade no processo foram: (a) contato por telefone e reunião presencial com a líder comunitária para apresentar o projeto e marcar o encontro com os demais moradores da comunidade; (b) encontro presencial com os moradores para apresentar o projeto e convidá-los a participar; (c) visitas às casas dos moradores da comunidade, a fim de entender os principais problemas relacionados à habitação. Importante ressaltar que o foco em habitação foi dado, em parte, pela participação de uma 
pequena empresa que fabrica móveis a partir da reciclagem de pallets, como 'patrocinador' da iniciativa.

A partir das informações coletadas, as análises realizadas pela equipe do NDS/UFPR resultaram em quatro problemas, cuja escolha se deu a partir de uma votação realizada com os moradores da comunidade. Esta votação ocorreu de maneira online e offline. A votação online foi conduzida com o auxílio de uma outra plataforma, a rede social Facebook, devido principalmente a dois fatores: (i) a plataforma innonatives.com, por ser uma plataforma internacional, disponibiliza todas as informações em inglês e na comunidade em questão as pessoas não tinham conhecimento deste idioma; (ii) pelas pessoas da comunidade em questão estarem familiarizadas com a rede social Facebook. Já a votação offline ocorreu através de uma urna levada pelos pesquisadores à comunidade. Como resultado, não obteve-se participação efetiva, apesar de ter sido realizada uma ação de comunicação como a entrega de panfletos e fixação de faixas na comunidade. Ao todo, a votação online recebeu apenas um voto. Já a votação offline recebeu 33 votos e foi considerada primordial na decisão da real necessidade.

Uma vez encontrada a real necessidade, lançou-se o desafio na plataforma innonatives, com a seguinte chamada: 'Can you design an artifact to improve the socialization in the kitchens of the low income houses?'. O processo foi delineado em três principais fases: ideação, conceituação e solução. As informações do briefing foram disponibilizadas em inglês e português, porém, a interface da plataforma innonatives.com (como botões e nomes das seções) somente apareciam em inglês.

Como estratégia de motivação à participação, além da premiação aos vencedores da melhor proposta de solução, foram realizados três workshops com estudantes de graduação em Design de duas Universidades, uma em Curitiba, PR, e outra em Joinville, SC. Tendo em vista que este tipo de iniciativa ainda é pouco conhecida no Brasil, os workshops presenciais foram importantes para esclarecer as dúvidas de participantes em potencial.

Assim, o desafio recebeu 26 ideias, oito conceitos e seis soluções. De acordo com a análise do desafio na plataforma, houve comentários em todas as fases, bem como em todas as propostas enviadas. A votação em cada uma das fases ocorreu de maneira online, sendo que a fase de escolha da melhor solução contou com a votação offline pela comunidade e votação através de um formulário enviado por e-mail aos especialistas. Na comunidade, a votação teve que ser realizada offline devido à razão apresentada anteriormente, ou seja, a restrição dos moradores da comunidade com relação à língua inglesa. A votação pelos especialistas também foi diferenciada pois a plataforma innonatives.com não possui uma função ativa com relação a mensuração da sustentabilidade das propostas enviadas - fator considerado primordial para a escolha da melhor solução, neste caso.

Com relação aos stakeholders envolvidos, bem como aos papéis e atividades por eles desempenhadas e a caracterização do tipo de envolvimento em offline ou online, é possível inferir que na iniciativa "The Kitchen Challenge":

- Além da participação dos três stakeholders fixos - ou seja, o ambiente online, o proponente da iniciativa e o resolutor de tarefas - houve o envolvimento de 'patrocinador', 'persona' e 'especialistas'. Ainda, aqueles que atuaram como o stakeholder 'resolutor de tarefas' desempenharam diferentes papéis, como 'criativo', 'comentarista' e 'votante'; 
- A participação dos stakeholders 'personas' foi mais eficaz de maneira offline. As tentativas de participação online foram limitadas devido às restrições com relação à língua estrangeira da plataforma, principalmente. Porém, a participação do stakeholder 'personas' foi fundamental para a escolha da necessidade e, portanto, para a definição do desafio;

- O stakeholder 'resolutor de tarefas', quando no desempenho do papel de 'criativo', foi envolvido na iniciativa tanto de maneira online como offline. A participação offline referese a participação em workshops presenciais ministrado pelo stakeholder 'proponente da iniciativa', ou seja, pelo grupo de pesquisadores do NDS/UFPR;

- Apesar de ser caracterizada como uma chamada aberta, ou seja, sem restrição de participantes, seja por habilidade ou conhecimentos específicos, notou-se que a participação nas 'tarefas criativas' foi, majoritariamente, de designers ou estudantes de design;

- A votação para a escolha da melhor solução ocorreu de maneira offline e online, sendo utilizados três diferentes procedimentos: (1) Votação offline na comunidade através de urna; (2) Votação online através da plataforma, pelos 'resolutores de tarefas' desempenhando o papel de 'votantes'; e (3) Votação online, porém fora da plataforma e através de um formulário específico, pelo stakeholder 'especialistas'.

Por fim, a implementação da solução não utilizou nenhuma das opções disponíveis na plataforma innonatives.com (a saber: crowdfunding, marketplace ou leilões). Ao invés disso, a proposta de melhor solução foi produzida pelo stakeholder 'patrocinador'. Um exemplar, então, foi sorteado na comunidade participante, como forma de agradecimento e reconhecimento pela participação na iniciativa.

\subsection{Caso 2: "Water for life"}

O segundo caso analisado foi o 'Water for Life', iniciativa de Crowd-Design também desenvolvida pelo NDS/UFPR e aplicada em uma empresa de grande porte ${ }^{3}$ localizada em Joinville, Santa Catarina. Neste caso, o início do processo se deu com a estruturação da iniciativa, ou seja, com a definição dos objetivos (briefing do desafio), de quem seriam os participantes, quais e quantas seriam as etapas. Para isso, a equipe de pesquisadores do NDS/UFPR se reuniu mais de uma vez com a equipe da empresa responsável pela iniciativa. Nesta etapa inicial, também foram envolvidos representantes dos setores jurídicos e de $\mathrm{RH}$, para consulta de questões legais e de pessoal da empresa.

Assim, pode-se dizer que a decisão do 'problema relacionado à sustentabilidade' a ser tratado pela iniciativa partiu de uma decisão consensual entre os responsáveis pelos setores da empresa envolvidos na iniciativa (setor de inovação, setor de RH e setor jurídico). Além desta adaptação na estruturação do processo em comparação à estruturação proposta pelo projeto Sustainability Maker, houve adaptações com relação ao tipo de participação necessária, visto que era a primeira vez que esta empresa estava aplicando uma iniciativa de Crowd-Design. Por

\footnotetext{
${ }^{3}$ Por motivos de contrato de sigilo, o nome da empresa não pode ser divulgado, bem como as informações referentes aos objetivos e resultados da iniciativa. As informações divulgadas, no entanto, referem-se ao processo de CrowdDesign e aos tipos de participação vinculados a este processo.
} 
exemplo, a empresa definiu que os participantes seriam seus próprios funcionários. Esta definição estratégica ocorreu porque, sendo uma empresa de grande porte, com unidades administrativas e fabris que somam mais de seis mil funcionários, em mais de 20 países, a empresa considerou cauteloso envolver apenas os funcionários do setor administrativo da sede em Joinville, SC; o que totaliza, aproximadamente, 500 funcionários. Ainda assim, mesmo sendo caracterizada como uma iniciativa de Crowd-Design interna, trata-se de uma iniciativa de inovação aberta, como visto anteriormente.

Outras adaptações necessárias foram: (1) redigir um regulamento interno, com definições específicas de participação dos funcionários na iniciativa; (2) intercalar as 'tarefas criativas' online com workshops presenciais (offline) de apoio ao desenvolvimento da proposta; (3) estipular uma premiação para motivar a participação, bem como recompensar os participantes da proposta de solução vencedora.

Assim, a iniciativa de Crowd-Design foi estruturada em quatro principais fases: (a) lançamento da iniciativa na empresa; (b) fase de ideação; (c) fase de conceituação; e (d) fase de solução. Estas três últimas são caracterizadas como 'tarefas criativas' e foram compostas por duas ações, cada uma: workshop presencial e envio da proposta (de ideia, conceito e solução) online.

Ao todo, foram 43 ideias, 29 conceitos e 10 soluções enviadas. Ao final, foram votadas três como as melhores soluções. A empresa optou por, após a fase de envio de conceito, limitar o número de propostas para 10 selecionadas para a fase seguinte.

Com relação aos stakeholders envolvidos, bem como aos papéis e atividades por eles desempenhadas e a caracterização do tipo de envolvimento em offline ou online, é possível inferir que na iniciativa 'Water for Life':

- Não fosse pelo papel de 'gerente', assumido pela empresa de grande porte em conjunto com o NDS/UFPR, haveria somente o envolvimento dos três stakeholders fixos (ambiente online, proponente da iniciativa e resolutor de tarefas);

- A empresa de grande porte foi a que desempenhou mais papéis, a saber: 'proponente da iniciativa', 'especialista', 'regulamentador' e 'patrocinador';

- O papel 'regulamentador' foi necessário devido às especificidades organizacionais (questões jurídicas e de $\mathrm{RH}$ ) da empresa de grande porte;

- Pelo fato do desafio ter sido definido pela própria empresa, ou seja, pelo stakeholder 'proponente da iniciativa', não houve a necessidade do papel 'persona';

- O envolvimento dos pesquisadores do NDS/UFPR foi caracterizado como 'gerente' pelo fato de estarem auxiliando a empresa na aplicação da iniciativa, visto que a empresa não possuía conhecimento específico em processos de Crowd-Design;

- A votação da proposta de melhor solução foi realizada online, mas também offline envolvendo especialistas da empresa de grande porte (no caso, diretores e gerentes dos setores de inovação e desenvolvimento de produtos).

Por fim, considera-se como implementação da proposta escolhida o fato da mesma ter sido incluída no processo de desenvolvimento de produtos da empresa. O que não significa, porém, que a proposta de melhor solução derivada da iniciativa será produzida de fato. Mas significa para a empresa uma inovação do processo de entrada do desenvolvimento de novos 
produtos.

\subsection{Categorização dos níveis de participação em iniciativas de Crowd-Design}

Num processo participativo como o Crowd-Design, não há um único tipo de participação, mas múltiplas possibilidades. Os níveis de participação, neste sentido, vão depender de como a iniciativa foi planejada e quais os objetivos da mesma. No entanto, por outro lado, nota-se que não há restrição de participação, a menos quando há o direcionamento explícito e proposital da participação de um público em especial (como foi o caso do "Water for Life').

Com relação ao meio onde a participação ocorre, ou seja, online ou offline, nota-se que em ambas iniciativas houve a necessidade de adotar os dois meios. Na iniciativa 'The Kitchen Challenge', a necessidade de realizar procedimentos offline deveu-se, principalmente, às características da comunidade envolvida. São elas: não familiaridade com o processo de CrowdDesign especificamente, falta ou restrição de acesso à internet $\mathrm{e}$, considerado o mais impeditivo à participação, o fato da plataforma innonatives.com estar em língua inglesa - idioma cuja maioria dos moradores da comunidade não domina ou não tem conhecimento. Já na iniciativa 'Water for Life', a necessidade de realizar workshops presenciais (e, por isso, offline) para estimular e desenvolver as habilidades criativas dos participantes deu-se pelo fato de este público ser considerado não-designer, ou seja, não possuíam conhecimento com o processo criativo.

Com base na figura 01 (apresentada na seção 2.2), os níveis de participação alcançados nestas duas iniciativas de Crowd-Design podem ser categorizados em (quadro 01):

Quadro 01 - Síntese dos resultados.

\begin{tabular}{lll}
\hline $\begin{array}{l}\text { Macro-etapas do } \\
\text { processo de } \\
\text { Crowd-Design }\end{array}$ & $\begin{array}{l}\text { Níveis de participação } \\
\text { no caso 'The Kitchen Challenge' }\end{array}$ & $\begin{array}{l}\text { Níveis de participação } \\
\text { no caso 'Water for Life' }\end{array}$ \\
\hline $\begin{array}{l}\text { DEFINIÇÃO DO } \\
\text { PROBLEMA }\end{array}$ & $\begin{array}{l}\text { A participação inicial se deu no nível de Consulta } \\
\text { Obrigatória, pois a votação para escolha do problema a } \\
\text { ser abordado foi essencial para o prosseguimento do } \\
\text { projeto. }\end{array}$ & $\begin{array}{l}\text { O início do projeto aconteceu com a } \\
\text { Informação dos projeto pela gerência aos } \\
\text { participantes do processo, sem consulta } \\
\text { aos mesmos. }\end{array}$ \\
\hline $\begin{array}{l}\text { CRIAÇÃO DE } \\
\text { SOLUÇÕES }\end{array}$ & $\begin{array}{l}\text { Nesta etapa a participação aconteceu no nível da } \\
\text { Facilitação da criação de soluções por meio de } \\
\text { Workshops offline ministrados pelos designers com } \\
\text { estudantes de graduação em Design do Paraná e de } \\
\text { Santa Catarina. }\end{array}$ & $\begin{array}{l}\text { Aqui os funcionários da empresa } \\
\text { participaram no nível da Facilitação, } \\
\text { participando de workshops presenciais de } \\
\text { estímulo à criatividade para geração de } \\
\text { soluções. }\end{array}$ \\
\hline
\end{tabular}

ESCOLHA DA

MELHOR

SOLUÇÃO
Aqui se deu o nível de participação de Co-design, pois usuários, designers e membros da comunidade puderam tomar a decisão de forma compartilhada.
Para a escolha da melhor solução, participaram no nível de Co-design funcionários, designers e gerentes.

Fonte: Dos autores (2017).

Por fim, estima-se que esta categorização, obtida a partir da aplicação da Abordagem Sistêmica das iniciativas de Crowd-Design apresentadas, possa representar um avanço no conhecimento tanto do processo de Crowd-Design quanto às possibilidades de participação nas diferentes etapas do processo. 


\section{Considerações finais}

Este artigo apresentou os resultados do estudo a respeito dos tipos e estratégias de participação utilizadas em iniciativas de Crowd-Design. A partir da aplicação da Análise Sistêmica como ferramenta de análise das informações coletadas pelos estudos de casos múltiplos ex-postfacto, foi possível categorizar a participação de stakeholders neste tipo de processo.

Considerando os três stakeholders fixos de uma iniciativa de Crowd-Design, foi possível perceber na prática que os stakeholders podem desempenhar mais de um papel, desenvolvendo mais de uma atividade. As 'tarefas criativas', apesar de serem abertas à participação de indivíduos da multidão que não precisam ter habilidades e conhecimentos específicos foram realizadas por indivíduos que possuíam algum conhecimento em design (no caso do 'The Kitchen Challenge') ou que foram estimulados e sentiram-se mais seguras ao receber algum tipo de suporte (no caso do 'Water for Life').

É importante que o stakeholder 'ambiente online' esteja preparado para propiciar as adaptações necessárias a cada iniciativa. Isso refere-se ao fato da plataforma innonatives.com ser uma plataforma internacional e que utiliza o inglês como idioma para comunicação em sua interface. Nesse sentido, observa-se a necessidade de desenvolvimento de uma plataforma de Crowd-Design que possa ser utilizada por pessoas no Brasil e que não tenham conhecimento da língua inglesa. Por um lado, isso significa restringir o acesso e participação às pessoas com conhecimento na língua portuguesa, por outro lado, significa a propiciar a inclusão da participação online de pessoas que não possuem conhecimento em língua estrangeira, como, por exemplo, o stakeholder 'personas' da iniciativa 'The Kitchen Challenge'.

Por fim, não foram abordados neste estudo aspectos relacionados à motivação para participação (sobre este assunto, consultar: Batistella \& Nonino, 2012, Simula \& Vuori, 2012; Simula \& Ahola, 2014; Geiger \& Schader, 2014). Porém, pode-se inferir que conhecer o que motiva a participação somado ao conhecimento sobre as possibilidades e estratégias de participação a serem utilizadas na iniciativa torna-se fundamental ao seu planejamento e consequentemente, ao seu sucesso.

\section{Referências}

BANNERMAN, S. Crowdfunding Culture. In: Journal of Mobile Media, v. 07, n. 01, p. 1-30, 2013.

BATTISTELLA, C.; NONINO, F. Open innovation web-based platforms: the impact of different forms of motivation on collaboration. In: Innovation Management Policy Practices, v. 14, n. 4, p. 557576, 2012.

BEHREND, T. S.; SHAREK, D. J.; MEADE, A. W. The viability of crowdsourcing for survey research. In: Behavior research methods, v. 43, n. 3, p. 800-813, 2011.

BORDENAVE, J. E. D. O que é participação. São Paulo: Editora Brasiliense, 1983.

BOTSMAN, R.; ROGERS, R. O que é meu é seu: Como o consumo colaborativo vai mudar o nosso mundo. Porto Alegre: Bookman, 2011.

BOUKHRIS, A. et al. Co-creation in the early stage of product-service system development. Procedia CIRP, vol. 63, p. 27-32. Julho, 2017.

BRABHAM, D. Crowdsourcing as a model for problem solving an introduction and cases. In: The international journal of research into new media technologies, v. 14, n.1, p.75-90, 2008.

. Crowdsourcing as a model for Problem Solving: leveraging the collective intelligence of Online Communities for public good. Thesis. Department of Communication The University of Utah, 2010. 
BROWN, T. Designers - Think big! TEDGlobal, jul. 2009. Disponível em:

<http://www.ted.com/talks/tim_brown_urges_designers_to_think_big> Acesso em 15 set. 2017. CABEZA, E. U. R.; MOURA, M. OPEN DESIGN: abertura + design = prática projetual para a transformação social. In: Anais do 110 Congresso Brasileiro de Pesquisa e Desenvolvimento em Design, v. 1, n. 4. São Paulo: Blucher, 2014.

$\mathrm{CHOI}$, J. H.; LEE, J. S. Online social networks for crowdsourced multimedia-involved behavioral testing: An empirical study. In: Frontiers in Psychology. 2015. Available at http:// dx.doi.org/10.3389/fpsyg.2015.01991.

CHIU, C. M.; LIANG, T. P.; TURBAN, E. What can crowdsourcing do for decision support? In: Decision Support Systems, v. 65, p. 40-49, 2014.

DAWSON, R.; BYNGHALL, S. Getting results from crowds. San Francisco: Advanced Human Technologies, 2012.

DE TONI, A. J.; BIOTTO, G.; BATISTELLA, C. Organizational design drivers to enable emergent creativity in web-based communities. In: The Learning Organization, v. 19, n. 4, p. 335-349, 2012. DICKIE, I.; SANTOS, A. Bases Para Entendimento Do Crowdsourcing E Sua Aplicação Em Projetos De Crowd-Design. In: Anais do 110 Congresso Brasileiro de Pesquisa e Desenvolvimento em Design, v. 1, n. 4. São Paulo: Blucher, 2014.

DJELASSI, S.; DECOOPMAN, I. Customers' participation in product development through crowdsourcing: Issues and implications. In: Industrial Marketing Management, v. 42, p. 683-692, 2013.

ESTELLES-AROLAS, E. A task-based crowdsourcing typology. In: Hansson et al. (Eds.), International Reports on Socio-Informatics (IRSI), Proceedings of the CSCW 2016 - Workshop: Toward a Typology of Participation in Crowdwork,v. 13, n. 1, pp. 57-64, 2016.

ESTELLES-AROLAS, E.; GONZALEZ-LADRON-DE-GUEVARA, F. Towards an integrated crowdsourcing definition. In: Journal of Information Science, v. 38, n. 2, p. 189-200, 2012.

FIEDLER-FERRARA, N. Quando o todo é mais sagaz do que a soma de suas partes. In:scientia zudia, São Paulo, v. 3, n. 2, p. 323-37, 2005.

GASSMANN, O.; DAIBER, M.; MUHDI, L. Der Crowdsourcing-Prozess. In: GASSMANN, O. (Edt.). Crowdsourcing - Innovationsmanagement mit Schwarmintelligenz. Carl Hanser Verlag München, p. 31-55, 2010.

GEIGER, D.; SEEDORF, S.; SCHULZE, T.; NICKERSON, R. C.; SCHADER, M. Managing the Crowd: Towards a Taxonomy of Crowdsourcing Processes. In: AMCIS 2011 Proceedings - All Submissions. Paper 430. 2011.

GEIGER, D.; SCHADER, M.. Personalized task recommendation in crowdsourcing information systems - Current state of the art. In: Decision Support Systems, v. 65, p. 3-16, 2014.

HOWE, J. The rise of crowdsourcing. In: Wired Magazine. 2006. Available at: http://www.wired.com/wired/archive/14.06/crowds.html. Accessed on January 2015.

IDEO.org Design Kit. Website. Disponível em: http://www.designkit.org/resources. Acesso em: novembro de 2017.

INNONATIVES PLATFORM. Plataforma online. Disponível em: https://www.innonatives.com/. Acesso em: outubro de 2017.

KAULIO, M. Customer, consumer and user involvement in product development: A framework and a review of selected methods. Total Quality Management, v. 9(1), p. 141-149, 1998.

KAZAZIAN, T. Haverá a idade das coisas leves: design e desenvolvimento sustentável. São Paulo: Senac, 2005. 
KRUCKEN, L. Competências para o design na sociedade contemporânea. In: Design e transversalidade. Belo Horizonte: Santa Clara: Centro de Estudos Teoria, Cultura e Pesquisa em Design, UEMG, 2008. (Cadernos de Estudos Avançado em Design, Caderno 2, v. 1 - jul. 2008), p.23-32.

LEGO IDEAS. Plataforma online. Disponível em: https://ideas.lego.com/dashboard. Acesso em: outubro de 2017.

MANZINI, E. Design para a inovação social e sustentabilidade: Comunidades criativas, organizações colaborativas e novas redes projetuais. Rio de Janeiro: Epapers, 2008.

. Social innovation and design - Enabling, replicating and synergizing. in: STEBBING, Peter e TISCHNER, Ursula. Changing Paradigms: Designing for a Sustainable Future. Aalto, Finlândia: Aalto University School of Arts, Design and Architecture, 2015.

MUKAZE, S.; VELÁSQUEZ, D. C. V. Product Service System: Co-Designing for Social Impact. Thesis (Master of Sustainable Product Service System Innovation) - School of Engineering, Blekinge Institute of Technology, Karlskrona, Sweden, 2012.

MUNIZ, M. O.; FIGUEIREDO, L. F. Redefinindo o design: por uma abordagem sistêmica. In: Actas de Diseño, 2007.

NAKATSU, R. T.; GROSSMAN, E. B.; IACOVOU, C. L. A taxonomy of crowdsourcing based on task complexity. In: Journal of Information Science, v. 40, n. 6, p. 823-834, 2014.

NO!SPEC. website. Disponível em: https://www.nospec.com/. Acesso em: outubro de 2017.

OPEN IDEO. Plataforma online. Disponível em: https://openideo.com/. Acesso em: outubro de 2017.

PENG, Z.; RUOYU, L. On operating mechanisms of crowdsourcing service innovation. In: IEEE, 2011. PRPI'C, J., SHUKLA, P. P., KIETZMANN, J. H., McCARTHY, I. P. How to work a crowd: Developing crowd capital through crowdsourcing. In: Business Horizons, v. 58, n. 1, p. 77-85, 2015.

SANDERS, E.; BRANDT, E.; BINDER, T. A Framework for Organizing the Tools and Techniques of Participatory Design. In: PDC 2010. Sydney, Australia.

SHARMA, A. Crowdsourcing Critical Success Factor Model Strategies to harness the collective intelligence of the crowd. 2010.2 Available at: https://irevolution.files.wordpress.com/2010/05/working-paper1.pdf. Accessed on: June 2015.

SIMULA, H. VUORI, M. Benefits and barriers of crowdsourcing in B2B firms: generating ideas with internal and external crowds. In: International Journal of Innovation Management, v. 16, n. 6, p. 1-19, 2012.

SIMULA, H.; AHOLA, T. A network perspective on idea and innovation crowdsourcing in industrial firms. In: Industrial Marketing Management, v. 43, p. 400-408, 2014.

STANOEVSKA-SLABEVA, K. Enabled Innovation: Instruments and Methods of Internet-based Collaborative Innovation. In: 1st Berlin Symposium in Internet and Society, 2011.

SUM (SUSTAINABILITY MAKER PROJECT). website. Disponível em: http://sustainabilitymaker.org/. Acesso em: outubro de 2017.

THACKARA, J. Plano B: o design e as alternativas viáveis em um mundo complexo. São Paulo: Saraiva, 2008.

THUAN, N. H.; ANTUNES, P.; JOHNSTONE, D. Factors influencing the decision to crowdsource: a systematic literature review. In: Inf. Syst. Front, v. 18, p. 47-68, 2016.

TISCHNER, U.; BESTE, L. State of the Art of Open Innovation and Design for Sustainability. In: MATSUMOTO et al. (Eds.), Sustainability Through Innovation in Product Life Cycle Design, EcoProduction: 2017. 